\title{
Advances in Therapeutic Plasma Exchange Technology and its Immunomodulatory Effect in Neuromuscular Diseases
}

\author{
An Expert Interview with Najib Khalife
}

Terumo BCT, Zaventem, Belgium

DOI: https://doi.org/10.17925/ENR.2019.14.2.61

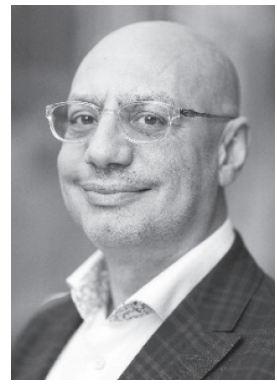

Najib Khalife

Najib Khalife is a Senior Manager of Global Medical Affairs at Terumo BCT. He is a medical doctor with 12 years' expertise in therapeutic apheresis and rare autoimmune diseases. He helped build the medical strategy and the medical affairs team across several regions, has facilitated multiple publications on therapeutic apheresis, and has been an invited speaker as subject matter expert on therapeutic plasma exchange at many medical society meetings and congresses.

\section{Keywords}

Immunomodulatory therapies, therapeutic plasma exchange, Guillain-Barré syndrome, myasthenia gravis, therapeutic apheresis

Disclosure: Najib Khalife is an ex-employee of Terumo BCT.

Review Process: This is an expert interview and, as such, has not undergone the journal's standard peer review process.

Compliance with Ethics: This article is an opinion piece and does not report on new clinical data, or any studies with human or animal subjects performed by the author.

Authorship: The named author meets the International Committee of Medical Journal Editors (ICMJE) criteria for authorship of this interview piece, takes responsibility for the integrity of the work as a whole, and has given final approval for the version to be published.

Access: This article is freely accessible at touchNEUROLOGY.com. ( ) Touch Medical Media 2020

Received: 5 November 2018

Published Online: 16 October 2019

Citation: European Neurological Review.

2019;14(2):61-3

Corresponding Author: Najib Khalife

Terumo BCT Europe, Ikaroslaan 41, 1930 Zaventem, Belgium. E: najibkhalife@gmail.com

Support: No funding was received in

the publication of this article.
$\mathrm{T}$ herapeutic plasma exchange (TPE) has been an accepted treatment for specific neurological disorders for several decades. For some medical professionals, it is seen as an effective treatment option alongside immunomodulatory therapies and other medicines. But its benefits for patients still need to be more widely recognised among medical professionals in many countries. Recent innovations in technology have made it an effective treatment option for a range of conditions, bringing patients more rapid results and increasing patient comfort. This interview discusses the recent developments in TPE, how this treatment has evolved in recent years and its benefits among other immunomodulatory therapies.

\section{Background}

The first English-language publication on plasma exchange, then described as plasmapheresis, was in 1914 by Abel et al. of Johns Hopkins University, where he performed the manual removal of a dog's blood, separated the plasma, and returned the cellular part to the animal. ${ }^{1}$ Since then the science of plasmapheresis has evolved progressively.

The first true milestone of partially automated plasma exchange was in the 1980s, with the introduction of apheresis devices based on centrifugation and filtration technologies. This revolutionised the science of what became known as "therapeutic plasma exchange". It also enabled researchers to investigate its immunomodulatory role in treating certain autoimmune diseases. At the same time, early clinical trials in plasma exchange for Guillain-Barré syndrome progressed, establishing TPE as the standard of care to treat patients suffering from this acute disease, bringing them fast recovery compared to supportive care. ${ }^{2-5}$ But it was not until the 1990s that immunoglobulins, which were initially used as replacement therapy for immunodeficiency patients, proved to be as effective a treatment as TPE for Guillain-Barré syndrome patients. ${ }^{6}$

For patients with myasthenia gravis, TPE is used in crisis situations of respiratory failure and before surgical removal of the thymus glands. TPE has been shown to shorten the time on mechanical ventilation, contributing to rapid patient response and recovery?

\section{Q. How does therapeutic plasma exchange work?}

TPE is different from other immunomodulatory therapies regarding technical treatment administration and mechanisms of action. Other immunomodulatory therapies are infused to patients and neutralise the circulating antibodies. During a TPE procedure, the patient's blood is separated by centrifugation into its components. The separated plasma, which contains circulating 
disease mediators, is removed and replaced by an appropriate type and equal volume of replacement fluid. ${ }^{8}$

TPE has been evaluated and is recommended by the European Academy of Neurology and by the Myasthenia Gravis Foundation of America as a safe and effective first-line therapy for patients with myasthenia gravis experiencing myasthenic crises and respiratory failure, and before surgical removal of the thymus gland (thymectomy). Moreover, TPE is considered for refractory patients with myasthenia gravis.

Various studies showed that by rapidly removing circulating disease mediators such as antibodies and cytokines, TPE has effectively and safely resulted in rapid recovery for the majority of patients with Guillain-Barré syndrome and myasthenia gravis. ${ }^{5,7}$

\section{Q. How are today's plasma exchange technologies different from plasmapheresis treatments of the past?}

continuous-flow centrifugation technology is a high-precision approach that has been refined over the past decade. It brings innovations in patient comfort and more efficient processing of blood and plasma products. One example is the Spectra Optia ${ }^{\circledR}$ Apheresis System (Terumo BCT Inc., Lakewood, CO, USA). It separates plasma from the other blood components in a highly efficient manner. ${ }^{9}$

This technique requires minimal blood flow compared with other technologies such as membrane separation approaches. It is an improved technology that allows easy access to blood through the patient's peripheral veins (antecubital or brachial), as compared with other membrane-based apheresis devices that mostly require invasive central venous catheterisation. These technical innovations also result in better patient comfort and safety during the TPE procedure than was previously possible and more accurate fluid balance control for better haemodynamic stability during treatment.10 Today's advanced plasma exchange systems use information tools and digital architecture that did not exist several years ago. These innovations contribute to enhanced procedural automation, user-friendly operation, data capturing and improved patient experience.

Continuous-flow centrifugation uses optical detection technologies with intelligent functions that offer a safe and standardised therapy to patients and ease-of-use to physicians, nurses and apheresis technicians. Features include precise and real-time monitoring of the position and thickness of separated blood - up to 25 times per second to 10 microns resolution. The built-in intelligent optical system (Automated Interface Management System) detects the formation of the buffy coat which contains platelets and white blood cells and returns it to the patient, sparing losses. Other useful management functions include continuous monitoring of fluid balance and automated adjustment of pump flow and valve function. The Spectra Optia Apheresis System applies this technology to accurately calculate the required dose of the exchanged plasma.

\section{Q. Does this mean that therapeutic plasma exchange replaces other immunomodulatory therapies and biologics to halt the progression of Guillain-Barré syndrome and myasthenia gravis?}

We know that these are complex conditions that manifest themselves at different severity levels. Guillain-Barré syndrome is a monophasic and self-limiting disease with seven clinical variants and ranges from mild to moderate and severe. TPE has been shown to shorten the disease course and rapidly improve the clinical outcome when administered at early onset of the disease. In this case, optimal results are observed when administered within 7-14 days of diagnosis.5 According to published evidence, corticosteroids are ineffective in treating Guillain-Barré syndrome; ${ }^{5}$ however, intravenous immunoglobulins (IVIG) are as effective as TPE. ${ }^{6}$ Recent publications show that patients with respiratory failure treated with TPE have less mechanical ventilator days than those treated with IVIG. ${ }^{11,12}$

Myasthenia gravis is a chronic disease with seven different subtypes, also ranging from mild to moderate and severe. Patients' myasthenia gravis crises are manifested by rapid respiratory failure and other acute neurological manifestations. TPE is used to treat patients with myasthenia gravis in crisis and pre-thymectomy states. Similarly, IVIG can be used to treat these two conditions. A growing body of evidence shows advantages of TPE over IVIG in certain patient subtypes, such as immunoglobulin G4-mediated muscle-specific tyrosine kinase (MuSK-Ab positive) ${ }^{13}$ and in juvenile myasthenia gravis.? Other biologics, such as pyridostigmine bromide, corticosteroids and immunosuppressive drugs, are used as maintenance therapies to treat mild and moderate disease conditions?

TPE rapidly removes circulating disease mediators, such as autoantibodies, immune complexes, and cytokines, resulting in improved recovery times compared with supportive care for the majority of patients with Guillain-Barré syndrome and myasthenia gravis. In one study of patients with myasthenia gravis, a TPE treatment course rapidly lowered all immunoglobulin levels. ${ }^{14}$

For myasthenia gravis, depending on disease severity and subtypes, the clinical approach is to use escalation therapies for acute and chronic disease states. IVIG and TPE are reserved for acute exacerbations, corticosteroids treat acute and chronic conditions, and immunosuppressive drugs are emerging maintenance therapies for chronic conditions. Monoclonal antibody drugs are more recent treatments for refractory myasthenia gravis. Methotrexate proved ineffective. ${ }^{15}$

\section{Q. What are the main areas where TPE can bring optimal comfort to patients and better performance to healthcare providers?}

Many specialised hospitals offer apheresis therapy as inpatient, and to a lesser extent as outpatient, services. A study of 42 patients with myasthenia gravis being treated with plasma exchange concluded that TPE therapy is safe, effective and well-tolerated. According to the authors, the study results do not raise concerns about the safety of TPE therapy in patients with moderate to severe myasthenia gravis. Ninety percent of patients were treated as outpatients and $83 \%$ were treated with peripheral vascular access. ${ }^{16}$

Another aspect contributing to patients' comfort is the timing and duration of the TPE procedure. An internal data analysis of 40,000 TPE procedures on the Spectra Optia System showed a median procedure time of 105 minutes per intervention. To achieve optimal patient response to TPE therapy, the timing of treatment must be considered. For Guillain-Barré syndrome it is suggested to initiate TPE within 7 days of onset for optimal response. ${ }^{5}$ For myasthenia gravis, expert consensus suggests that TPE is more effective and works more quickly than IVIG in impending or manifest myasthenic crises.

Current experience shows that TPE is generally safe and well-tolerated by patients. ${ }^{17}$ The majority of reactions to the therapy are mild, easily 
treated and of limited duration. According to the World Apheresis Association registry data of adverse events in apheresis, for 16,000 apheresis procedures: $94.2 \%$ of patients who underwent the procedure did not experience adverse events, 1.6\% had mild reactions, 3.8\% showed moderate reactions, and $0.7 \%$ had severe reactions. ${ }^{17}$ The issue of difficult venous access is a concern for some patients, and it has been noted that severe adverse events are higher with central access procedures compared to peripheral access. Of 1,207 TPE procedures tracked, $79.3 \%$ were conducted via peripheral access. ${ }^{18}$

For some hospitals, TPE has demonstrated significant cost savings. A US cost-minimisation study showed a cost of $\$ 10,000$ for five IVIG infusion sessions, compared with $<\$ 4,700$ for five TPE procedures. ${ }^{19}$

Based on a meta-analysis of 15 publications on TPE cost effectiveness compared with IVIG, and assuming equality in terms of patient characteristics and hospital type, TPE is a cost-effective therapy when compared with $\mathrm{VIIG}^{20}$ In terms of the total treatment costs, savings between $15-83 \%$ are possible when TPE is applied compared with IVIG. Health systems that foresee an increased use of TPE versus IVIG in the treatment of Guillain-Barré syndrome are likely to benefit from cost savings. ${ }^{20}$

\section{Additional Information}

\section{Spectra Optia Apheresis System}

The Spectra Optia system is an industry-leading therapeutic apheresis device for TPE, cell processing, cell collection and other apheresis procedures. It allows operators to spend more time focusing on patient care rather than attending procedure operation. This advanced system uses continuous-flow centrifugation, automation and optical detection technology that allows operators to perform a wide variety of apheresis procedures on a single platform. The Spectra Optia System is the first, and only, device on the European market today that has received the CE mark with an indication to treat Guillain-Barré syndrome and myasthenia gravis. ${ }^{9}$

\section{Therapeutic plasma exchange with the Spectra Optia Apheresis System}

- Provides automated monitoring and allows for the accurate maintenance of fluid balance

- Helps efficiently remove circulating disease mediators and achieve highly predictable results with the patented Automated Interface Management System

- Has a plasma removal efficiency* of up to $87 \%{ }^{10}$

- Offers flexibility to perform a single- or dual-needle peripheral vascular access, and both inpatient and outpatient procedures

- Accommodates and supports patient comfort and safety

- For some 40,000 procedures, the median procedure time was 105 minutes

*Plasma removal efficiency is an established metric to analyse the performance of an apheresis device during a TPE procedure.
1. Abel JJ, Rowntree LG, Turner BB. On the removal of diffusible substances from the circulating blood by means of dialysis.
Transactions of the Association of American Physicians, 1913 . Transactions of the Association

2. Färkkilä M, Kinnunen E, Haapanen E, Livanainen M. Guillain Barré syndrome: quantitative measurement of plasma exchange therapy. Neurology. 1987;37:837-40.

3. Greenwood RJ, Newsom-Davis J, Hughes RAC, et al. Controlled trial of plasma exchange in acute inflammatory polyradiculoneuropathy. Lancet. 1984;1:877-9.

4. Osterman PO, Fagius J, Lundemo G, et al. Beneficial effects of plasma exchange in acute inflammatory polyradiculoneuropathy. Lancet. 1984;2:1296-9.

5. Chevret S, Hughes RAC, Annane D. Plasma exchange for Guillain-Barré syndrome. Cochrane Database Syst Rev. 2017;2:CD001798

6. Hughes R. Randomised trial of plasma exchange, intravenous immunoglobulin, and combined treatments in Guillain-Barré syndrome. Plasma Exchange/Sandoglobulin Guillain-Barré syndrome. Plasma Exchange/Sandoglobulin Guilai

7. Sanders DB, Wolfe GI, Benatar M, et al. International consensus guidance for management of myasthenia gravis: executive summary. Neurology. 2016;87:419-25
8. Reeves HM, Winters JL. The mechanisms of action of plasma exchange. Br J Haematol. 2014;164:342-51.

. Cision PR B nswire System is the First and On BCT's Spectra Optia Apheresis System Is the First and Only Device on the European Market Indicated to Treat Guillain-Barre Syndrome and Myasthenia Gravis using Therapeutic Plasma Exchange, 2017. Available at: www. prnewswire.co.uk/news-releases/terumo-bctsspectra-optia-apheresis-system-is-the-first-and-only-deviceon-the-european-market-618465043.html (accessed 4 September 2019).

10. Tormey CA, Peddinghaus ME, Erickson M, et al. Improved plasma removal efficiency for therapeutic plasma exchange using a new apheresis platform. Transfusion. 2010;50:471-7.

11. El-Bayoumi MA, El-Refaey AM, Abdelkader AM, et al. Comparison of intravenous immunoglobulin and plasma exchange in treatment of mechanically ventilated children with Guillain-Barré syndrome: a randomized study. Critical Care. 2011,15:R164

12. Saad K, Mohamad IL, Abd El-Hamed M, et al. A comparison between plasmapheresis and intravenous immunoglobulin in children with Guillain-Barré syndrome in Upper Egypt. Ther Adv Neurol Disord. 2016;9:3-8
13. Pasnoor $\mathrm{M}$, Wolfe $\mathrm{G}$, Nations $\mathrm{S}$, et al. Clinical findings in MuSK-antibody positive myasthenia gravis: a U.S. experience. Muscle Nerve. 2010;41:370-4.

14. Guptill JT, Juel VC, Massey JM, et al. Effect of therapeutic plasma exchange on immunoglobulins in myasthenia gravis. plasma exchange on immunog

15. Pasnoor $\mathrm{M}, \mathrm{He} \mathrm{J}$, Herbelin L, et al. A randomized controlled trial of methotrexate for patients with generalized myasthenia gravis. Neurology. 2016;87:57-64

16. Ebadi $H$, Barth $D$, Bril V. Safety of plasma exchange therapy in patients with myasthenia gravis. Muscle Nerve. 2013;47:510-4.

17. Mörtzell Henriksson $M$, Newman $E$, Witt $V$, et al. Adverse events in apheresis: an update of the WAA registry data. Transfus Apher Sci. 2016;54:2-15.

18. Noseworthy JH, Shumak KH, Vandervoort MK. Long-term use of antecubital veins for plasma exchange. Transfusion. 1989:29:610-3.

19. Winters JL, Brown $D$, Hazard E, et al. Cost minimization analysis of the direct costs of TPE and IVIG in the treatment of Guillain-Barré syndrome. BMC Health Serv Res. 2011:11:101

20. Dierick K. Latest insights on the cost efficiency of therapeutic plasma exchange in treating Guillain-Barré syndrome. Value Health. 2018;21(Suppl. 1):S166. 